\title{
Explicit and Semi-implicit complex diffusion schemes for Optical Coherence Tomography despeckling
}

\author{
Pedro Rodrigues ${ }^{1}$, Pedro Guimarães ${ }^{2}$, Adérito Araújo ${ }^{3}$, Silvia Barbeiro ${ }^{3}$, \\ Rui Bernardes ${ }^{1,2}$, and Pedro Serranho ${ }^{1,4}$ \\ ${ }^{1}$ Centre of New Technologies for Medicine, Association for Innovation and \\ Biomedical Research on Light and Image \\ Azinhaga de Santa Comba, Celas, 3000-548 Coimbra, Portugal; \\ 2 Institute of Biomedical Research in Light and Image, Faculty of Medicine, \\ University of Coimbra \\ Azinhaga de Santa Comba, Celas, 3000-548 Coimbra, Portugal; \\ 3 CMUC, Department of Mathematics, University of Coimbra, \\ Apartado 3008, 3001-454 Coimbra, Portugal; \\ 4 Mathematics Section, Department of Science and Technology, Open University \\ Campus TagusPark, Av. Dr. Jacques Delors, 2740-122 Porto Salvo, Oeiras, Portugal.
}

\begin{abstract}
In this paper we illustrate the use of explicit and semiimplicit finite difference schemes for nonlinear complex diffusion in the context of medical imaging despeckling, namely for Optical coherence Tomography images. Differente boundary conditions will also be used. Performance metrics are shown to illustrate the feasibility of the numerical schemes and compare its results.
\end{abstract}

Keywords: Optical Coherence Tomography, denoising, despeckling, complex diffusion, finite differences

\section{Introduction}

Diffusion processes are commonly used for image processing in general, such as impaiting, denoising and stereo vision [3, 4,6-8,10-12]. Particular applications for image denoising and despeckling are of interested $[3,5,7,8]$ and have been used in the past decade. The these methods are usually based in the discretization of a nonlinear diffusion equation of the form

$$
\frac{\partial u}{\partial t}(x, t)=\nabla \cdot(D(x, t, u) \nabla u(x, t)), \quad \text { in } Q \times[0, T]
$$

where the solution $u(x, t)$ represents different stages of the filtered image, $x$ is the spatial coordinate defined in the square $Q=\left[1, N_{1}\right] \times\left[1, N_{2}\right]$ (where the inital image as dimensions $N_{1} \times N_{2}$ ), $t$ is the time coordinate defined in the interval $[0, T]$ where $T$ is the diffusion time, and the diffusion coefficient $D$ has to be properly defined in order to avoid diffusion across intensity edges in the 
image and therefore a blurring effect. Perona and Malik [7] proposed to use a diffusion coefficient based in the gradient of the image, in order to distinguish between edges and constant regions. However, in the initial stpes od the image where the noise level is high, the gradient is unstable. To overcome this problem, Gilboa [5] suggested to consider an appropriate complex filter of the form

$$
D=\frac{e^{i \vartheta}}{1+\left(\frac{\operatorname{Im}(u)}{\kappa \vartheta}\right)^{2}}
$$

where $\vartheta \approx 0$ and $\kappa$ is a positive coefficient. It was proven that this filter is efficient, since

$$
\lim _{\vartheta \rightarrow 0} \frac{\operatorname{Im}(u(., t))}{t \theta}=G * \Delta I_{0}
$$

where $I_{0}$ is the initial image, $G$ is a gaussian and $*$ holds for the convolution operator. In [3], the method was adapted for the case o Optical coherence tomography (OCT) images. In [1] rigorous stability results were proven for finite difference schemes for (1) with complex diffusion coefficient. The convergence result was also achieved in [2].

Though implicit and semi-implicit finite differences methods are shown to be unconditionally stable, engineers tend to implement and use explicit schemes for the diffusion process. The explicit scheme is easier to implement, but the step in time is limited by the stability condition, leading to the need of a higher number of time steps required. On the other hand, implicit and semi-implicit schemes need to solve a linear system at every time step, though the number of time steps can be considerably lower.

In this work we will compare explicit and semi-implicit schemes for complex diffusion of OCt images in terms of image metrics. In this way, we aim at showing how these versions perform in terms of denoising metrics. Moreover, we will consider both Dirichlet and Neumann boundary conditions for the equation (1), in order to illustrate if it has an effect on the denoising of the image.

The paper is organized as follows. In section 2 we present the numerical schemes and the considered boundary conditions. In section 3 we present the main results, showing denoising metrics of each condidered approach. Finally, we draw some conclusions and future perspectives in section 4 .

\section{Methods}

Let $I_{0}$ be the original (noisy) image of size $N_{1} \times N_{2}$. Complex diffusion is usually based in the numerical solution of the partial differential equation (1). In order to have a well posed problem, equation (1) must be complemented by an initial condition of the form

$$
u(x, 0)=I_{0},
$$

where $I_{0}$ is the original (noisy) image. Moreover, one needs boundary conditions, defined in the boundary $\Gamma$ of the set $Q$. We will consider Dirichlet boundary 
conditions

$$
u(x, t)=g(x, t), \quad x \in \Gamma, t \in[0, T],
$$

which means that the boundaries of the image are kept fixed with some given $g$. In alternative, we will also consider Neumann boundary conditions

$$
\frac{\partial u}{\partial \nu}(x, t)=0, \quad x \in \Gamma, t \in[0, T]
$$

which means the there is no normal intensity flux, where $\nu$ is the unit exterior normal vector.

\subsection{Discretization}

We consider an equally spaced mesh on $Q$. Let $h_{1}=h_{2}=1$ be the mesh spacement in the first and second spatial coordinate direction. The mesh is therefore defined by the set of points

$$
x_{j, k}=(j, k), \quad i=0,1,2, \ldots, N_{1}+1, k=0,1,2, \ldots, N_{2}+1 .
$$

Moreover, we consider the a time spacement $h_{t, m}$ which defines the set of points

$$
t_{m+1}=t_{m}+h_{t, m}, \quad m=0,1, \ldots, N_{t} .
$$

such that $t_{N_{t}}=T$. Thefore we define a mesh $Q_{h}$ defined by the set of points

$$
\left(x_{j, k}, t_{m}\right), \quad i=0,1,2, \ldots, N_{1}+1, k=0,1,2, \ldots, N_{2}+1, m=0,1, \ldots, N_{t} .
$$

We consider the general finite difference scheme (see eq.(2.9) in [1]) for the differential equation (1) given by

$$
\begin{aligned}
U_{j, k}^{m+1}=U_{j, k}^{m}+\frac{h_{t, m}}{2 h_{1}^{2}}[ & \left(D_{(j+1, k)}^{m, \theta, \mu}+D_{(j, k)}^{m, \theta, \mu}\right) U_{(j+1, k)}^{m+\theta}+\left(D_{(j, k)}^{m, \theta, \mu}+D_{(j-1, k)}^{m, \theta, \mu}\right) U_{(j-1, k)}^{m+\theta} \\
& \left.-\left(D_{(j+1, k)}^{m, \theta, \mu}+2 D_{(j, k)}^{m, \theta, \mu}+D_{(j-1, k)}^{m, \theta, \mu}\right) U_{(j, k)}^{m+\theta}\right] \\
+\frac{h_{t, m}}{2 h_{2}^{2}}[ & \left(D_{(j, k+1)}^{m, \theta, \mu}+D_{(j, k)}^{m, \theta, \mu}\right) U_{(j, k+1)}^{m+\theta}+\left(D_{(j, k)}^{m, \theta, \mu}+D_{(j, k-1)}^{m, \theta, \mu}\right) U_{(j, k-1)}^{m+\theta} \\
& \left.-\left(D_{(j, k+1)}^{m, \theta, \mu}+2 D_{(j, k)}^{m, \theta, \mu}+D_{(j, k-1)}^{m, \theta, \mu}\right) U_{(j, k)}^{m+\theta}\right]
\end{aligned}
$$

for $j=1,2, \ldots, N_{1}, k=1,2, \ldots, N_{2}, m=0,1, \ldots, N_{t}-1$ where $U_{j, k}^{m}$ is the approximation to the solution $u\left(x_{j, k}, t_{m}\right)$,

$$
D_{(j, k)}^{m, \theta, \mu}=D\left(x_{j, k}, t^{m+\theta}, U_{j, k}^{m+\mu}\right)
$$

and $\mu \in\{0,1\}, t^{m+\theta}=\theta t^{m+1}+(1-\theta) t^{m}, \theta \in[0,1]$ and

$$
V_{j, k}^{m+\theta}=\theta V_{j, k}^{m+1}+(1-\theta) V_{j, k}^{m}, \quad V=U, D,
$$


Several choices of $(\theta, \mu)$ give several different numerical schemes.

We will consider the explicit scheme given by $\theta=0$ and $\mu=0$, that is,

$$
\begin{aligned}
U_{j, k}^{m+1}= & {\left[1-\frac{h_{t, m}}{2 h_{1}^{2}}\left(D_{(j+1, k)}^{m}+2 D_{(j, k)}^{m}+D_{(j-1, k)}^{m}\right)\right.} \\
& \left.-\frac{h_{t, m}}{2 h_{2}^{2}}\left(D_{(j, k+1)}^{m}+2 D_{(j, k)}^{m}+D_{(j, k-1)}^{m}\right)\right] U_{j, k}^{m} \\
+ & \frac{h_{t, m}}{2 h_{1}^{2}}\left[\left(D_{(j+1, k)}^{m}+D_{(j, k)}^{m}\right) U_{(j+1, k)}^{m}+\left(D_{(j, k)}^{m}+D_{(j-1, k)}^{m}\right) U_{(j-1, k)}^{m}\right] \\
+ & \frac{h_{t, m}}{2 h_{2}^{2}}\left[\left(D_{(j, k+1)}^{m}+D_{(j, k)}^{m}\right) U_{(j, k+1)}^{m}+\left(D_{(j, k)}^{m}+D_{(j, k-1)}^{m}\right) U_{(j, k-1)}^{m}\right]
\end{aligned}
$$

for $j=1,2, \ldots, N_{1}, k=1,2, \ldots, N_{2}, m=0,1, \ldots, N_{t}-1$. This scheme is known to be stable (see [1]) for

$$
h_{t, m} \leq \frac{\min \left(h_{1}, h_{2}\right)}{4 \max \frac{\left|D_{j, k}^{m}\right|^{2}}{\operatorname{Re}\left(D_{j, k}^{m}\right)}}
$$

We will also consider the semi-implicit scheme given by $\theta=1$ and $\mu=0$. This scheme is known to be unconditionally stable (see [2]), so one can choose a fixed step in time $h_{t}=T / N_{t}$, that is, one as the numerical scheme

$$
\begin{aligned}
& {\left[1+\frac{h_{t}}{2 h_{1}^{2}}\left(D_{(j+1, k)}^{m}+2 D_{(j, k)}^{m}+D_{(j-1, k)}^{m}\right)\right.} \\
& \left.\quad+\frac{h_{t}}{2 h_{2}^{2}}\left(D_{(j, k+1)}^{m}+2 D_{(j, k)}^{m}+D_{(j, k-1)}^{m}\right)\right] U_{j, k}^{m+1} \\
& -\frac{h_{t}}{2 h_{1}^{2}}\left[\left(D_{(j+1, k)}^{m}+D_{(j, k)}^{m}\right) U_{(j+1, k)}^{m+1}+\left(D_{(j, k)}^{m}+D_{(j-1, k)}^{m}\right) U_{(j-1, k)}^{m+1}\right] \\
& -\frac{h_{t}}{2 h_{2}^{2}}\left[\left(D_{(j, k+1)}^{m}+D_{(j, k)}^{m}\right) U_{(j, k+1)}^{m+1}+\left(D_{(j, k)}^{m}+D_{(j, k-1)}^{m}\right) U_{(j, k-1)}^{m+1}\right] \\
& =U_{j, k}^{m},
\end{aligned}
$$

for $j=1,2, \ldots, N_{1}, k=1,2, \ldots, N_{2}, m=0,1, \ldots, N_{t}-1$. For this numerical schemme, a linear system needs to be solved at each iteration. As an iterative method is advised (for computation efficiency) we consider the preconditioned conjugate gradient algorithm of MatLab.

One also needs to consider the initial condition

$$
U_{j, k}^{0}=I_{0}(j, k), \quad j=1,2, \ldots, N_{1}, k=1,2, \ldots, N_{2} .
$$

We also consider the Dirichlet boundary condition

$$
\begin{array}{cc}
U_{j, 0}^{m}=I_{0}(j, 1), U_{j, N_{2}+1}^{m}=I_{0}\left(j, N_{1}\right), & j=1,2, \ldots, N_{1}, \\
U_{0, k}^{m}=I_{0}(1, k), U_{N_{1}+1, k}^{m}=I_{0}\left(N_{1}, k\right), & k=1,2, \ldots, N_{2},
\end{array}
$$


for $m=0,1, \ldots, N_{t}$ or the Neumann condition

$$
\begin{array}{cl}
U_{j, 0}^{m}=U_{j, 2}^{m}, U_{j, N_{2}+1}^{m}=U_{j, N_{2}-1}^{m}, & j=1,2, \ldots, N_{1}, \\
U_{0, k}^{m}=U_{2, k}^{m}, U_{N_{1}+1, k}^{m}=U_{N_{1}-1, k}^{m}, & k=1,2, \ldots, N_{2},
\end{array}
$$

In this work we will compare the performances of the following numerical schemes:

1. Explicit method with Dirichlet boundary conditions defined by the system of equations (7), (9), (10);

2. Explicit method with Neumann boundary conditions defined by the system of equations (7), (9), (11);

3. Semi-implicit method with Dirichlet boundary conditions defined by the system of equations (8), (9), (10);

4. Semi-implicit method with Neumann boundary conditions defined by the system of equations (8), (9), (11);

\subsection{Diffusion coefficient}

We consider the complex diffusion coefficient (2) proposed by Gilboa [5]. While Gilboa considered $\kappa$ constant, an improved version for OCT filtering was proposed in [3] as

$$
\kappa=\kappa_{M I N}+\left(\kappa_{M A X}-\kappa_{M I N}\right) \frac{g-\min (g)}{\max (g)-\min (g)}
$$

where $\kappa_{M I N}, \kappa_{M A X}$ are given real constants with $\kappa_{M I N}<\kappa_{M A X}$ and

$$
g=G_{N, \sigma} * \operatorname{Re}(u)
$$

where $G_{N, \sigma}$ is a gaussian of size $N \times N$ and standard deviation $\sigma$ and $*$ holds for the convolution operator.

\subsection{Performance metrics}

To test the performance of the proposed filtering methods, we consider both synthetic and real OCT images.

In the synthetic images [9], one creates a noisefree synthetic OCT image and adds OCT-like speckle noise. In this way it makes sense to use metrics like the mean square error (MSE)

$$
\mathrm{MSE}=\frac{\sum_{j=1}^{N_{1}} \sum_{k=1}^{N_{2}}\left(I(i, j)-U_{j, k}^{N_{t}}\right)^{2}}{N_{1} N_{2}}
$$

where $I$ is noisefree image, and the mean structure similarity index [?]

$$
M S S I M=\frac{1}{N} \sum_{j, k} S S I M\left(v_{I}(j, k), v_{U}(j, k)\right)
$$


with

$$
\operatorname{SSIM}\left(v_{1}, v_{2}\right)=\frac{\left(2 \mu_{1} \mu_{2}+C_{1}\right)\left(2 \sigma_{12}+C_{2}\right)}{\left(\mu_{1}^{2}+\mu_{2}^{2}+C_{1}\right)\left(\sigma_{1}^{2}+\sigma_{2}^{2}+C_{2}\right)}
$$

where $v_{I}(j, k)$ and $v_{U}(j, k)$ represent local windows of the images $I$ and $U^{N_{t}}$, respectively, in the neighborhood of the coordinate $(j, k), C_{1}=(0.01 L)^{2}, C_{2}=$ $(0.03 L)^{2}$ and $L$ is the maximum value allowed for the data (e.g., 255 for 8-bit data). The values $\mu_{j}, \sigma_{j}$ and $\sigma_{12}$ are given by

$$
\begin{aligned}
\mu_{j} & =\sum_{k} w(k) v_{j}(k) \\
\sigma_{j} & =\sqrt{\sum_{k} w(k)\left(v_{j}(k)-\mu_{j}\right)^{2}} \\
\sigma_{12} & =\sum_{k} w(k)\left(v_{1}(k)-\mu_{1}\right)\left(v_{2}(k)-\mu_{2}\right)
\end{aligned}
$$

with $j=1,2$ and $w$ a trivariate Gaussian weight function (of integral equal to one and standard deviation 1.5) [?].

For both sinthetic or real OCT filtered images we also computed the effective number of looks (ENL), the signal to noise ratio (SNR) and the contrast to noise ratio $(\mathrm{CNR})$ given by

$$
\mathrm{ENL}=\frac{\mu_{H}^{2}}{\sigma_{H}^{2}}, \quad \mathrm{SNR}=\frac{\mu_{H}}{\sigma_{H}}, \quad \mathrm{CNR}=10 \log \left(\frac{\mu_{R}-\mu_{H}}{\sqrt{\sigma_{R}^{2}+\sigma_{H}^{2}}}\right),
$$

where $H$ is an homogeneous (background) region and $R$ is a feature region in the image.

\section{Results}

We present the results in table 1 . There are no significant differences in the filtering performance between the explicit and semi-implicit schemes, nor between the Neumann and Dirichlet boundary condition. There are significant differences for OCT filtering between $\kappa$ constant or $\kappa$ adaptive, has previously shown for the explicit Dirichlet case [3].

\section{Conclusion}

The performance in terms of filtering seems to be independent of the numerical scheme (explicit or semi-implicit) or the bounday condition (non-homogeneous Dirichelet or homogeneous Neumann). In this way, one can choose the best scheme in terms of computation eficiency. At this moment, this comparison has not yet been done, since the linear solver for the semi-implicit scheme can still be optimized. 
Table 1. Metrics for the considered numerical schemes, using $\kappa$ constant and adaptive.

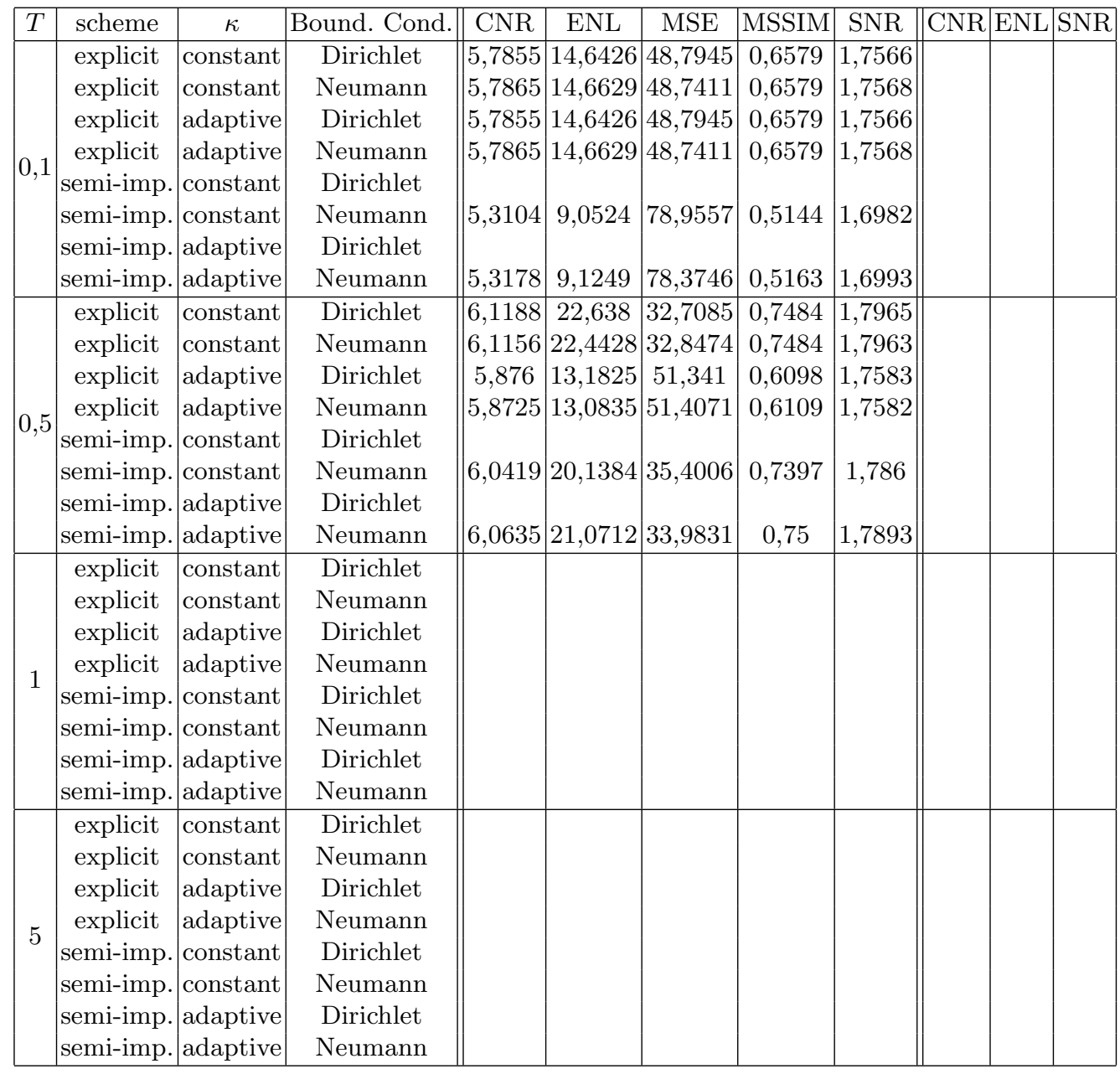


We have left the implicit method defined by $\theta=1$ and $\mu=1$ outside the scope of this comparison, since it needs a nonlinear solver at each step. The choice of the linearization process for the solver influences the result and will be object of future research.

Acknowledgment. P. Rodrigues, P. Guimarães, R. Bernardes and P. Serranho acknowledge the support by FCT under the research projects PTDC/SAUBEB/103151/2008 and PTDC/SAU-ENB/111139/2009, and by the COMPETE programs FCOMP-01-0124-FEDER-010930 and FCOMP-01-0124-FEDER-015712. A. Araújo and S. Barbeiroackowledge the partial support by CMUC and FCT through European program COMPETE/FEDER and project UTAustin/MAT/0066/2008.

\section{References}

1. Araújo, A., Barbeiro, S., Serranho, P.: Stability of finite difference schemes for complex diffusion processes. SIAM J. Numer. Anal. 50 (3), 1284-1296 (2012)

2. Araújo, A., Barbeiro, S., Serranho, P.: Finite difference schemes for nonlinear complex diffusion processes. to appear (2013?)

3. Bernardes, R., Maduro, C., Serranho, P., Araújo, A., Barbeiro, S., Cunha-Vaz, J.: Improved adaptive complex diffusion despeckling filter. Optics Express 18 (23), 24048-24059 (2010)

4. Brox, T., Bruhn, A., Papenberg, N., Weickert, J.: Computer Vision - ECCV 2004. Lecture Notes in Computer Science, vol. 3024, chap. High accuracy optical flow estimation based on a theory for warping, pp. 25-36. Springer, Berlin (2004)

5. Gilboa, G., Sochen, N., Zeeni, Y.: Image enhancement and denoising by complex diffusion processes. IEEE Trans Pattern Anal Mach Intell 26 (8), 1020-1036 (2004)

6. Grossauer, H., Scherzer, O.: Scale Space Methods in Computer Vision, Lecture Notes in Computer Science, vol. 2695, chap. Using the Complex Ginzburg-Landau Equation for Digital Inpainting in 2D and 3D, pp. 225-236. Springer (2003)

7. Perona, P., Malik, J.: Scale-space and edge detection using anisotropic diffusion. IEEE Trans Pattern Anal Mach Intell 12 (7), 629-639 (1990)

8. Salinas, H., Fernández, D.: Comparison of PDE-based nonlinear diffusion approaches for image enhancement and denoising in optical coherence tomography. IEEE Trans. Med. Imaging 26 (6), 761-771 (2007)

9. Serranho, P., Maduro, C., Santos, T., Cunha-Vaz, J., Bernardes, R.: Synthetic oct data for image processing performance testing. In: International Conference in Image Processing (ICIP) 2011. pp. 401-404 (2011)

10. Weickert, J.: Anisotropic diffusion filters for image processing based quality control. Proc. 7th Eur. Conf. Mathematics in Industry 1252, 355-362 (1994)

11. Weickert, J.: A review of nonlinear diffusion filtering. In: ter Haar Romeny, B.M., Florack, L., Koenderink, J.J., Viergever, M.A. (eds.) Scale-Space. Lecture Notes in Computer Science, vol. 1252, pp. 3-28. Springer (1997)

12. Zimmer, H., Bruhn, A., Valgaerts, L., Breuß, M., Weickert, J., Rosenhahn, B., Seidel, H.P.: Vision, Modeling, and Visualization, chap. PDE-based anisotropic disparity-driven stereo vision, pp. 263-272. AKA Heidelberg (2008) 